\title{
Quantitative and qualitative comparison of DNA amplification by PCR with immunofluorescence staining for diagnosis of Pneumocystis carinii pneumonia
}

\author{
T R Leigh, B G Gazzard, A Rowbottom, J V Collins
}

\begin{abstract}
Aim: To compare the results of DNA amplification by the polymerase chain reaction (PCR) with immunofluorescence staining for detecting Pneumocystis carinii in bronchoalveolar lavage specimens taken from symptomatic HIV seropositive patients with suspected $P$ carinii pneumonia (PCP).

Methods: Bronchoalveolar lavage specimens were obtained from 28 symptomatic HIV seropositive patients. Specimens were examined for $P$ carinii using immunofluorescence, and by DNA amplification with PCR to obtain results on gel electrophoresis (gel) and a more sensitive Southern hybridisation (blot) technique. Specimens positive by immunofluorescence and gel electrophoresis were serially diluted to a $10^{-6}$ concentration and each dilution strength tested for $P$ carinii using PCR to compare quantitatively immunofluorescence with PCR.
\end{abstract}

Results: Of the 28 specimens analysed, 18 were negative for $P$ carinii by both immunofluorescence and PCR, two were positive only by the blot technique of PCR, four were equivocally positive and four unequivocally positive by immunofluorescence. Three of the four equivocally positive patients tested by immunofluorescence were negative for $P$ carinii by PCR, although one was positive by PCR (blot) technique. This patient had clinically confirmed PCP. Of the four unequivocally positive patients tested by immunofluorescence, three were gel and blot positive by PCR and had PCP clinically, but one was negative by both gel and blot techniques, although the patient certainly had PCP on clinical grounds. This patient had received nine days of treatment with high dose co-trimoxazole before bronchoalveolar lavage specimens were obtained. The three specimens positive by gel and blot techniques remained gel positive down to dilutions of between $10^{-4}$ and $10^{-6}$.

Conclusions: PCR results may become negative soon after starting treatment for PCP. Specimens should therefore be taken before, or soon after, starting treatment. PCR seems to be between $10^{4}$ and $10^{6}$ times more sensitive than immunofluorescence.

(F Clin Pathol 1993;46:140-144)
Pneumocystis carinii pneumonia (PCP) affects $75 \%$ of patients with AIDS and remains the most common presenting defining illness in patients infected with the human immunodeficiency virus (HIV). ${ }^{1}$ In spite of the routine use of chemoprophylaxis, PCP still carries a mortality of between $12 \%$ and $33 \%$, depending on the experience of individual institutions for treating such patients. ${ }^{2}$ Detection of $P$ carinii infection has been hampered in the past by our inability to grow the organism in vitro, and until recently has relied on microscopic visualisation of the organism in suitably stained specimens. ${ }^{3}$ Several different stains have been successfully used, but the Grocott silver stain was traditionally the $m$ : st popular, and remained so until the development in 1989 of a new monoclonal antibody based immunofluorescence stain. ${ }^{4}$ This immunofluorescence stain was subsequently shown to be more sensitive than the pre-existing stains, ${ }^{5}$ but doubt has been cast on its specificity, with reports of false positive or equivocal (less than five cysts) results. ${ }^{6}$ In spite of this, however, immunofluorescence has been adopted in several institutions for the routine diagnosis of $P$ carinii infection in patients with AIDS in place of the traditional silver stain.

The recent successful cloning of a mitochondrial ribosomal RNA gene specific to $P$ carinii has now resulted in a new highly st 2 sitive test for diagnosing $P$ carinii infection, using DNA amplification by the polymerase chain reaction (PCR). ${ }^{78}$ This PCR technique is mainly a research tool at present, but it has now been shown to be more sensitive than immunofluorescence for diagnosing $P$ carinii infection, ${ }^{9}$ although a direct quantitative cumparison of the sensitivities of the $t w$ techniques has not yet been performed. This study sets out to compare quantitatively and qualitatively the sensitivity of these two techniques.

\section{Methods}

Over a five month period $28 \mathrm{HIV}$ positive patients (mean age 37 years, mean CD4 lymphocyte count $31 \times 10^{9} / 1$ ) each underwent fibreoptic bronchoscopy with bronchoalveolar lavage. The patients were a consecutive series of symptomatic individuals presenting with cough, dyspnoea, or unexplained pyrexia, undergoing investigation for suspected PC.P. All subjects had previously undergone sputurn induction, a highly sensitive technique for 


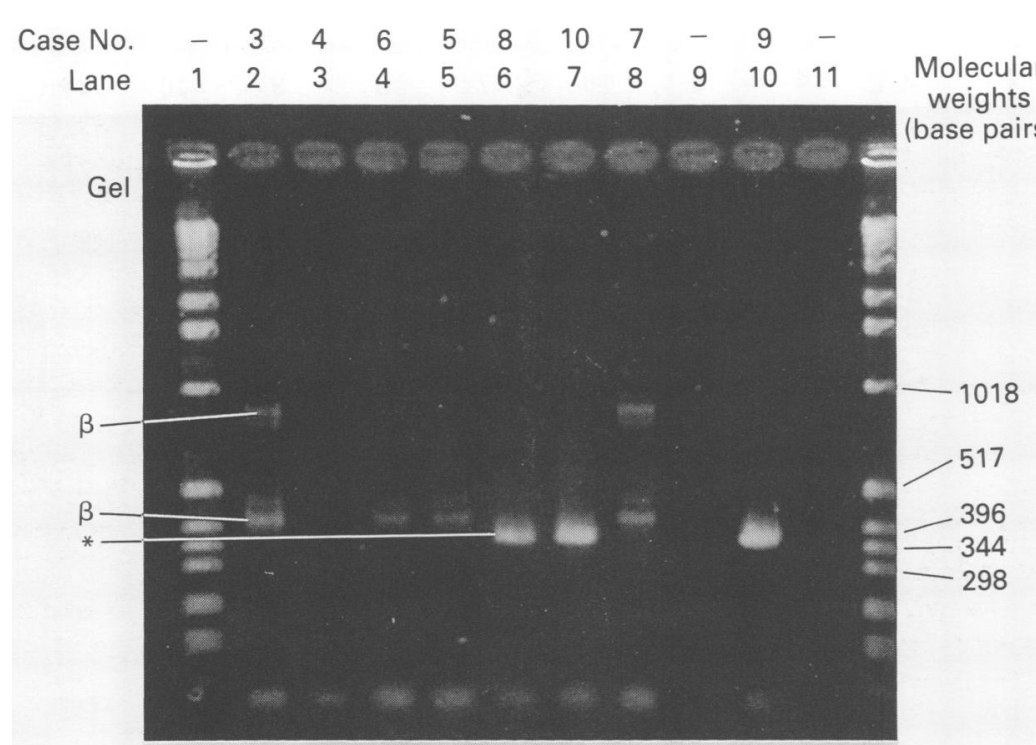

Oligoblot

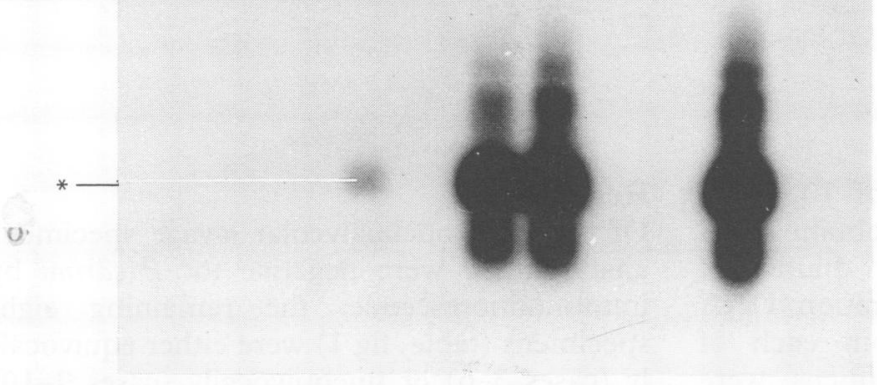

* Level of $P$ carinii specific PCR product.

$\beta$ Position of the non-specific PCR products

Figure 1 Gel electrophoresis and oligoblot results from the immunofluorescence positive and immunofluorescence equivocal patients. The asterisk points to the PCR product specific to $P$ carinii. The $\beta$ points to the non-specific PCR products. diagnosing PCP in patients with AIDS, ${ }^{10}$ with a negative result for $P$ carinii by immunofluorescence staining. (Patients with positive results received treatment for PCP and did not require fibreoptic bronchoscopy). Twenty three of the 28 subjects were receiving chemoprophylaxis for PCP at the time of the bronchoscopy. All specimens were examined for the presence of $P$ carinii using two techniques, an immunofluorescence stain (Northumbria Biologicals, UK) and DNA amplification using PCR. The immunofluorescence stain utilises a murine monoclonal antibody to the cyst wall of $P$ carinii, as described by Albert et al. ${ }^{11}$

\section{DNA AMPLIFICATION FOR DETECTION OF P CARINII}

The oligonucleotide primers pAZ102-E (sequence: 5'-G-A-T-G-G-C-T-G-T-T-T-CC-A-A-G-C-C-C-A-3') and pAZ102-H (sequence: 5'-G-T-G-T-A-C-G-T-T-G-CA-A-A-G-T-A-C-T-C-3') were used to amplify $P$ carinii DNA as described previously. ${ }^{7}$ A positive control was included using extracted DNA from a case of PCP that had been confirmed by both silver and immunofluorescence stains, as were negative controls with no added template DNA. The PCR products were electrophoresed on $1.5 \%$ agarose gels and visualised by ethidium bromide staining to give a gel result. The $P$ carinii specific PCR product appears as a discrete band corresponding to a molecular weight of 346 base pairs, as predicted by sequencing studies, ${ }^{7}$ and is indicated by an asterisk in figs 1 and 2 . Also seen in the gel are two non-specific PCR products. These are indicated by a $\beta$ symbol in fig 1 ; it is believed that these result from annealing of the primers to chance identical (or near identical) human genomic DNA sequences which are also present in the sputum specimen, but which are easily distinguished from the $P$ carinii specific PCR product by their different (slower) mobility in the gel. These non-specific products, indicated in fig 1 by the molecular weight scale next to the 1 kilobase molecular weight ladder (Gibco BRL, Uxbridge), have molecular weights of about 396 and 1000 base pairs, respectively, and their presence confirms successful DNA extraction from the sputum specimen, and successful DNA amplification, but they do not indicate the presence of $P$ carinii. In specimens strongly positive for $P$ carinii, these non-specific products are either absent or greatly reduced in quantity as a result of the preferential consumption of the primers and nucleotides by the $P$ carinii specific DNA sequence (lanes 6,7 , and 10 in fig 1).

Gel electrophoresis is followed by oligonucleotide hybridisation after Southern transfer (oligoblotting) with a ${ }^{32} \mathrm{P}$ end-labelled probe, pAZ102-L2 (sequence: 5'-A-T-A-A-G-G-TA-G-A-T-A-G-T-C-G-A-A-A-G-3'), (an oligomer internal to the $P C R$ primers) to give a blot result, which at high stringency $\left(48^{\circ} \mathrm{C}\right)$ is specific for human derived $P$ carinii and does not bind to the non-specific PCR products described above. ${ }^{7}$ Filters were autoradiographed at $-80^{\circ} \mathrm{C}$ for 16 hours or four days to show up particularly weak bands of $P$ carinii.

Thus PCR has two levels of sensitivity for the detection of $P$ carinii, a less sensitive gel result, and a more sensitive (by about 100 times) autoradiograph blot result able to detect as few as one organism in each PCR reaction mixture. ${ }^{12}$ DNA extraction and PCR preparation were performed in a laminar flow cabinet to avoid cross-contamination.

\section{QUANTIFICATION OF RELATIVE SENSITIVITIES} OF PCR WITH IMMUNOFLUORESCENCE All specimens which were found to be either positive, or equivocally positive for $P$ carinii by immunofluorescence were used to compare quantitatively the relative sensitivities of the two techniques. The extracted DNA template material from these specimens was 
Figure 2 Gel electrophoresis of PCR products from serial dilutions of Case 9. Dilutions range from $10^{\circ}$ $10^{-6}{ }^{*}$ Asterisk shows level of $P$ carinii specific PCR product.

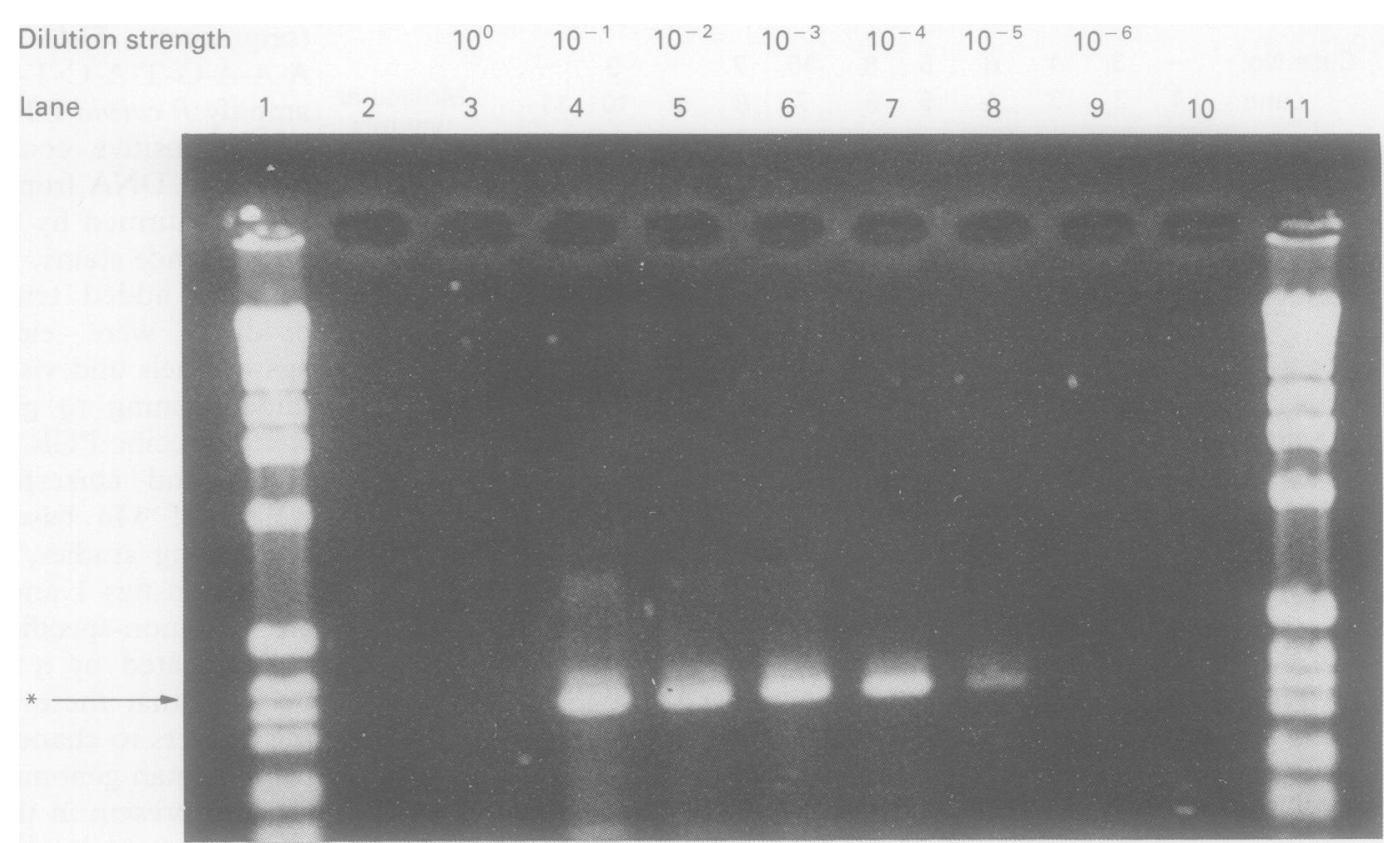

sequentially diluted by powers of 10 , using sterile double distilled water to obtain DNA template dilutions down to a $10^{-6}$ dilution of the full strength $(\times 1)$ concentration DNA template material. Aliquots from each of these sequentially diluted specimens were then used as DNA template material to be amplified by the polymerase chain reaction to detect the presence of $P$ carinii, ${ }^{7}$ and visualised by both gel electrophoresis and autoradiography. For comparison, an identical dilution protocol was also performed on an induced sputum specimen positive for $P$ carinii by immunofluorescence. In this way a critical level of dilution was established in order to obtain a positive PCR result on gel electrophoresis. This enabled a back calculation to be made of the organism load required for a positive immunofluorescence result, as well as an estimation of the relative sensitivity of immunofluorescence with PCR.
Patients positive or equivocally positive for $P$ carinii by either immunofluorescence or PCR

\begin{tabular}{|c|c|c|c|c|}
\hline $\begin{array}{l}\text { Case } \\
\text { No }\end{array}$ & $\begin{array}{l}\text { Immunofluorescence } \\
\text { result } \pm \text { or }+ \text { or - }\end{array}$ & $\begin{array}{l}\text { PCR result } \\
\text { gel or blot }\end{array}$ & $\begin{array}{l}\text { Duration/type } \\
\text { of treatment }\end{array}$ & $\begin{array}{l}\text { Clinical } \\
\text { outcome }\end{array}$ \\
\hline 1 & - & Blot & $4 / 7$ & \multirow{6}{*}{$\begin{array}{l}\text { Recovery } \\
\text { Recovery } \\
\text { Pulmonary } \\
\text { Kaposi's sarcoma/ } \\
\text { recovery } \\
\text { Recovery } \\
\text { Pulmonary } \\
\text { Kaposi's sarcoma/ } \\
\text { recovery } \\
\text { Recovery }\end{array}$} \\
\hline 2 & - & Blot & & \\
\hline 3 & \pm & - & $\begin{array}{l}\text { 4/7 Primaquine } \\
\text { and clindomycin }\end{array}$ & \\
\hline 4 & \pm & - & 4/7 Co-trimoxazole & \\
\hline 5 & \pm & - & 1/7 Co-trimoxazole & \\
\hline 6 & \pm & Blot & $\begin{array}{l}\text { 2/7 Primaquine and } \\
\text { cindomycin }\end{array}$ & \\
\hline 7 & + & 一 & 9/7 Co-trimoxazole & \multirow{4}{*}{$\begin{array}{l}\text { HIV } \\
\text { encephalopathy } \\
\text { Recovery } \\
\text { Recovery } \\
\text { Died }\end{array}$} \\
\hline 8 & + & gel + blot & 4/7 Co-trimoxazole & \\
\hline 9 & + & gel + blot & 3/7 IV pentamidine & \\
\hline 10 & + & gel + blot & 4/7 Co-trimoxazole & \\
\hline
\end{tabular}

^Before bronchoalveolar lavage.

\section{Results}

Of the 28 bronchoalveolar lavage specimens analysed, 20 were negative for $P$ carinii by immunofluorescence, the remaining eight specimens (table, fig 1 ) were either equivocally (cases 3-6) or unequivocally (cases 7-10) immunofluorescence positive, and received a full two week course of high dose treatment for PCP. When the 20 immunofluorescence negative specimens were examined for $P$ carinii using PCR, 18 were negative by both gel and blot techniques, but two were positive only by the more sensitive blot technique, demonstrating a low level of $P$ carinii colonisation. Of these 20 immunofluorescence negative individuals, 12 of whom had received treatment for PCP, all made uneventful recoveries from their respiratory illnesses, and none developed PCP during a six month follow up period.

Of the four specimens equivocally positive by immunofluorescence (cases 3-6), three were negative for $P$ carinii by both gel and blot techniques (cases 3-5) with only non-specific PCR products visible on the gel (indicated by a $\beta$ symbol in fig 1 ) and no signal on the oligoblot, and one was positive by blot alone (case 6) (table, fig 1) with only non-specific products visible on the gel, but with a low level signal from the $P$ carinii specific PCR product seen on the oligoblot (lower section of fig 1). Cases 3 and 5 were diagnosed endoscopically as having endobronchial Kaposi's sarcoma, and case 6 almost certainly had PCP on clinical grounds, with pronounced interstitial shadowing on the chest radiograph, exercise induced arterial oxygen desaturation, an arterial $\mathrm{PO}_{2}$ concentration on room air of $6.2 \mathrm{kPa}$ and a good clinical response to primaquine and clindomycin. Case 4 was receiving dapsone as primary PCP chemoprophylaxis and had a CD4 count of $8 \times 10^{9} /$; chest radiograph 
and arterial blood gases were both normal. All four patients had received between one and four days of high dose treatment for PCP before having the bronchoscopy and all subsequently recovered from their acute respiratory illnesses.

Of the four specimens which were unequivocally immunofluorescence positive (cases 7 and 10), one was negative for $P$ carinii by both the gel and blot techniques (case 7) with only non-specific products visible on the gel and no signal on the oligoblot, and three were both gel and blot positive on PCR (cases 8-10) with bright $P$ carinii specific PCR products on the gel at the 346 base pair position (indicated by an asterisk in fig 1), and strong signals centred at a corresponding position on the oligoblot. Two of the three gel and blot positive patients (cases 8 and 9) responded to high dose treatment for PCP, but the remaining patient (case 10) did not respond and subsequently died. The one immunofluorescence positive patient negative for $P$ carinii by both gel and blot techniques (case 7) was felt to have PCP on clinical grounds, with diffuse interstitial shadowing on the chest radiograph, and arterial hypoxia. He had been treated with high dose treatment for PCP for nine days before the bronchoscopy with only partial improvement, and subsequently developed a toxic confusional state, a cause for which was never fully established. Ultimately a clinical diagnosis of HIV encephalopathy was made.

\section{QUANTIFICATION STUDIES}

The three specimens which were both gel and blot positive for $P$ carinii using PCR (cases 8-10) were used for the quantification studies. Case 8 gave a positive gel result down to a dilution of $10^{-4}$ of the full strength $(\times 1)$ DNA template material; cases 9 (fig 2) and 10 gave positive gel results down to dilutions of $10^{-5}$ and $10^{-6}$, respectively. By comparison, the extracted DNA template specimen prepared from induced sputum, gave a positive gel result down to a dilution of $10^{-3}$.

\section{Discussion}

Although the specimen numbers presented here are small, this is the first study to show an anomaly between the results obtained by DNA amplification and immunofluorescence in the diagnosis of $P$ carinii infection. In the only previous study of DNA amplification for detecting $P$ carinii, it was shown that active or incipient PCP was invariably associated with a positive gel and blot result, and that no patient positive by conventional staining was gel negative by PCR. ${ }^{6}$ It is interesting to note, therefore, that in the present study there were several specimens that gave discrepant immunofluorescence and PCR results when compared with the clinical diagnoses. One example of this was a patient who was unequivocally positive by immunofluorescence, but was found to be negative on PCR by both the gel and blot techniques (case 7). This could represent either a false positive immunofluorescence result, or, alternatively, a false negative PCR result. From a clinical perspective the diagnosis was almost certainly PCP: the chest radiograph appearance was typical of PCP, with interstitial shadowing, and severely impaired blood gas exchange. We must conclude, therefore, that this was most probably a false negative PCR result. One possible explanation for this, that the specimen contained an inhibitor of the PCR reaction, is excluded by the presence of nonspecific PCR products on the electrophoresis gel. The second explanation may lie in the fact that the bronchoalveolar lavage specimen from this patient was taken nine days after starting high dose treatment for PCP, compared with a maximum of four days for cases 8-10 (table 1). $P$ carinii cysts are demonstrable with conventional stains for up to a month after starting treatment, ${ }^{13}$ but similar studies have not yet been performed using PCR, and the previous study ${ }^{9}$ which showed PCR to be more sensitive than immunofluorescence was performed on transplant recipients receiving no chemoprophylaxis. In the previous study using PCR for detection of $P$ carinii ${ }^{6}$ no information was given regarding the prior use of treatment for PCP. When compared with the cyst wall, as detected by immunofluorescence and silver stains, the $P$ carinii specific DNA detected by PCR could quickly disappear once specific treatment for PCP is begun. This would result in a rapid conversion from a positive to negative PCR result. Similarly, case 6 who had PCP on clinical grounds received two days of high dose treatment for PCP before having bronchoalveolar lavage. This may have been sufficient time for the PCR result to have changed from a gel and blot positive result (as predicted by the previous study ${ }^{6}$ ) to a blot positive result alone. If this hypothesis proves correct PCR may be a useful measure of organism viability and therefore of treatment response, as has been recently shown for cytomegalovirus infection in bone marrow transplant recipients. ${ }^{14}$ The three negative PCR results (cases 3-5) with equivocal immunofluorescence results may, in the light of preceding discussions, be either due to prior chemotherapy, or represent false positive immunofluorescence results. Given that the patients had only received between one and four days of treatment before bronchoscopy, which was probably insufficient time for the PCR result to become completely negative, these probably represent false positive immunofluorescence results.

The dilution studies described here assume that the quantity of PCR product produced by the polymerase chain reaction is directly proportional to the quantity of DNA template material in the reaction mixture. This is an approximation frequently adopted by researchers, but nevertheless makes the quantitative comparison of immunofluorescence with PCR only an estimate of their relative sensitivities. We would have liked to perform similar dilution studies with the immunofluorescence stain but this was not possible on 
these specimens. We feel, however, that this omission is partly offset by the fact that none of the specimens was strongly positive by immunofluorescence, and that all patients had previously been negative for $P$ carinii by sputum induction, suggesting a relatively low level of $P$ carinii organism load. PCR (gel) seems, therefore, to be between $10^{4}$ and $10^{6}$ times more sensitive than immunofluorescence for detecting $P$ carinii in bronchoalveolar lavage specimens. As it has been shown that a positive gel result requires the presence of about 7500 organisms $/ \mathrm{ml}$ of bronchoalveolar lavage from which the DNA has been extracted, ${ }^{12}$ this suggests that the limit of sensitivity of immunofluorescence by extrapolation is about $75 \times 10^{6}$ to $75 \times 10^{8}$ organisms $/ \mathrm{ml}$ of bronchoalveolar lavage specimen, and less for induced sputum. This would correlate with organism loads observed in other clinically important pulmonary infections. ${ }^{15}$ It is also interesting to note that the patient who died had the highest organism load (case 10).

TRL is supported by a grant from the North West Thames Regional Health Authority.

1 Murray JF, Mills J. Pulmonary infectious complications of human immunodeficiency virus infection. Part 2. Am Rev Respir Dis 1990;141:1582-98.

2 Bennett CI, Gertler P, Guze PA, Garfinkle JB, Kanouse $D E, G r e e n f i e l d ~ S$. The relation between resource use and in-hospital mortality for patients with acquired and in-hospital mortality for patients with acquired immunodeficiency syndrome-related Pneumocystis carinii pneumonia. Arch Intern Med 1990;150:1447-52.
Masur H, Lane HC, Kovacs JA, Allegra CJ, Edman JC. Pneumocystis pneumonia: From bench to clinic. Ann
Int Med 1989;111:813-26.

4 Dournon E, Rajagopalan P, Albert F, et al. Diagnosis of Pneumocystis carinii pneumonia by non-experts. Lancet 1989;333:107.

5 Leigh TR, Parsons P, Midgley J, Husain OAN, Shanson DC, Collins JV. Immunofluorescent staining improves the diagnostic sensitivity of sputum induction in Pneumocystis carinii pneumonia. Thorax 1989;44: 10:891P.

6 Leigh TR, Parsons P, Midgley J, et al. Increased sensitivity of immunofluorescence for detection of Pneumocystis carinii. Lancet 1989;334:1523.

7 Wakefield AE, Pixley FJ, Banerii S, et al. Amplification of mitochondrial ribosomal RNA sequences from Pneumocystis carinii DNA of rat and human origin. Mol Biochem Parasitol 1990;43:69-76.

8 Wakefield AE, Pixley FJ, Banerii S, et al. Detection of Pneumocystis carinii with DNA amplification. Lancet 1990;336:451-53.

9 Leigh TR, Wakefield AE, Peters SE, Hopkin JM, Collins JV. Comparison of DNA amplification and immunofluorescence for detecting Pneumocystis carinii in patients receiving immunosuppressive therapy. Transplantation 1992;54:468-70.

10 Leigh TR, Parsons P, Hume C, Husain OAN, Gazzard BG, Collins JV. Sputum induction for diagnosis of pneumocystis carinii pneumonia. Lancet 1989;334: 205-6.

11 Albert F, McLauchlin J, Taylor AG, Rajagopalan P, Darnon L. Detection of Pneumocystis carinii cysts in broncho-alveolar lavage fluids from AIDS patients using a monoclonal antibody. Communicable Disease Report. a monoclonal

12 Peters SE, Wakefield AE, Banerji S, Hopkin JM. Quantification of the detection of Pneumocystis carini by DNA amplification. Mol Cell Probes 1992;6:115-17.

13 Shelhamer JH, Ognibene FP, Macher AM, et al. Persistence of Pneumocystis carinii in lung tissue of acquired immunodeficiency syndrome patients treated for Pneumocystis pneumonia. Am Rev Respir Dis 1984; 130:1161-5.

14 Einsele H, Ehninger G, Steidle M, et al. Polymerase chain reaction to evaluate antiviral therapy for cytomegalo reaction to evaluate antiviral therapy
virus disease. Lancet 1991;338:1170-2.

15 Currie DC, Higgs E, Metcalfe S, Roberts DE, Cole PJ. A simple method of monitoring the colonising microbial load in chronic bronchial sepsis: Pilot comparison of the reduction in colonising microbial load with intermittent and continuous antibiotics. F Clin Pathol 1987; 40:830-6. 